\title{
Effect of Cytochrome P450 2C19 681G>A Polymorphism on Premature Coronary Heart Disease
}

\author{
Zhong-Hai Chi, Yuan-Yuan Li, Juan Li, Zheng Zhang*, Li Jia \\ The Affiliated Hospital of Medical College Qingdao University \\ Qingdao, China
}

\begin{abstract}
Objective: The aim of our study was to evaluate the association between cytochrome P450 2C19 (CYP2C19) 681G>A polymorphisms and the age of development of coronary heart disease. Additionally, the study might find some biological indicators at the gene level that could help to predict and evaluate the risk of coronary heart disease in time.

Methods: This study included 352 individuals with coronary heart disease. Polymerase chain reaction-restriction fragment length polymorphism (PCR-RFLP) was used to identify CYP2C19 681G>A. The objects were divided into wild type (GG or homozygous $C Y P 2 C 19 * 1$ wild-type) and mutant type (GA/AA or the mutant CYP2C19*2 allele) on the basis of genotype. The association between CYP2C19 gene polymorphism and the age of onset of coronary heart disease was assessed by multivariate linear regression analysis.

Results: There was a significant association in the age of onset between the two groups $(\mathrm{t}=3.398, P=0.001)$, which was $58.51 \pm 12.72$ years in the wild type and $53.95 \pm 11.63$ years in the mutant group. The frequency of CYP2C19 681A $\left(C Y P 2 C 19 * 2\right.$ allele) was $0.32,0.268,0.227$ in different age groups, which was significantly different $\left(\chi^{2}=10.745, P=0.005\right)$ in different groups, as well as in the genotype. The result, assessed by multiple linear regression, showed that genotype, smoking, obesity, and hyperlipidemia affect the age of onset of coronary heart disease ( $\beta$ was $-0.167,0.156,0.155$, and $0.112, P<0.05)$, and mutually affect the age of onset of coronary heart disease.

Conclusion: The study suggests that CYP2C19 $681 \mathrm{G}>\mathrm{A}$ gene polymorphism may be one of the risk factors in susceptibility to early onset of coronary heart disease, but not an independent factor because other factors may play a synergistic role.
\end{abstract}

Key words: CYP2C19 $681 G>A$ polymorphism; premature coronary heart disease; age of onset.

\section{Introduction}

In recent years, coronary heart disease (CHD) has caused a rise in mortality and morbidity, and serious harm to human life and health. Moreover, the trend in morbidity has been gradually toward younger people. The incidence of coronary heart disease is closely related to genetic and environmental factors, particularly premature CHD (age of onset $<55$ years in males, and $<65$ years in females) but is more likely to be influenced by genetic factors. At present, a large number of clinical and statistical studies have indicated that (CYP2C19) $681 \mathrm{G}>\mathrm{A}$ polymorphism can affect drug metabolism associated with CHD and the prognosis for patients with the disease. However, research focusing on the effects of the incidence of

*Corresponding author: Zheng Zhang, Graduate student from Medical College Qingdao University; Qingdao, China. E-mail: 163zhangz@163.com
CHD are still rare. Therefore, this study, with the onset age as the breakthrough point, was to investigate the correlation between (CYP2C19) 681G $>$ A polymorphism and CHD and further explore the role of $\mathrm{CYP} 2 \mathrm{C} 19$ gene polymorphism normality in CHD.

As one of the key microsomal mixed function oxidases, the Cytochrome P450 enzymes (CYPs) play an important role in oxidation, peroxidation, and reduction of endogenous physiological substances, such as steroid hormones and cholesterol metabolism, as well as in maintaining cardiovascular homeostasis. The polymorphic P450 (CYP) enzyme superfamily is the most important system involved in the biotransformation of many endogenous and exogenous substances including drugs, toxins, and carcinogens. Genotyping for CYP polymorphisms provides important genetic information that help to understand the effects of xenobiotics on human body. Its high gene polymorphism is one of the earliest pharmacogenetics research objects [1]. 
While CYP2 is the largest family, of which CYP2C19 is a member, its genetic polymorphism is the gene encoding mutation causing the enzyme's abnormal activity, and has visible differences in different populations. Even in different ethnic groups in China, there are obvious differences between the incidence of wild type and mutant-type alleles [2]. Now, a number of studies have found that CYP2C19 gene polymorphism is significantly associated with the risk and prognosis of coronary heart disease. Ecan et al. [3] found that heterozygous CYP2C19*3 allele frequency in patients with coronary artery stenosis is significantly higher than in the control group ( $10.2 \%$ and $5.6 \%$, respectively), suggesting that the mutation can significantly increase the risk of CHD.

\section{Methods}

\section{$\underline{\text { Study population }}$}

Three hundred and fifty-two patients with coronary heart disease (according to The European Society of Cardiology Pocket Guide) were enrolled in the study from January 2013 to August 2014, all of whom were from the Han nationality in northern China. Individuals with the following conditions were removed from this study: serious heart disease, chronic hepatic or kidney disease, malignant tumors, acute and chronic infection, and recent major surgery. This research had the approval of the ethics committee and subjects signed informed consent.

\section{Study protocol}

Peripheral venous blood was drawn from each patient after fasting for at least 10 hours, and was tested for serum total cholesterol (TC), triglyceride (TG), high density lipoprotein (HDL), and low density lipoprotein (LDL) in half an hour. The tests were implemented by a Roche Hitachi 7600 automatic biochemistry analyzer in our hospital's biochemical laboratory. Professionals measured the height and weight of all subjects, calculating BMI=body weight $(\mathrm{kg}) /[\text { height }(\mathrm{m})]^{2}$. Overweight means a body mass index $>25 \mathrm{~kg} / \mathrm{m}^{2}$, according to the standard diagnosis that was announced by WHO in 1997. All subjects needed to provide medical history, including smoking, history of diabetes, hypertension, and hyperlipidemia. Diabetes means a fasting blood glucose $>7.0 \mathrm{mmol} / \mathrm{L}$ and (or) postprandial blood glucose $\geq 11.1 \mathrm{mmol} / \mathrm{L}$ or having a history of hypoglycemic treatment. Hypertension means systolic pressure $\geq 140 \mathrm{mmHg}$ and (or) diastolic blood pressure $90 \mathrm{mmHg}$ or having a history of antihypertensive treatment. Any one of the following conditions, orreceivinglipid-lowering therapy, canbediagnosed as hyperlipidemia: $\mathrm{TC} \geq 5.72 \mathrm{mmol} / \mathrm{L}, \mathrm{TG} \geq 1.70 \mathrm{mmol} / \mathrm{L}$, LDL$\mathrm{C} \geq 3.64 \mathrm{mmol} / \mathrm{L}$, and HDL-C $\geq 0.91 \mathrm{mmol} / \mathrm{L}$. According to the smoking standardization recommendations issued by WHO in 1984, smoking means that one smokes 1 cigarette or more per day and has done so for more than 1 year.

\section{$\underline{\text { Genetic analysis }}$}

For our study, 2 to $3 \mathrm{ml}$ of peripheral venous blood was drawn from every individual. The CYP2C19 genotype was detected by a BaiO-BE gene chip detector, a BE-2.0 biological chip reading instrument, PCR amplification detection, and finally the result was automatically determined by the Array Doctor analysis software (genotype was completed by Shanghai United gene company). The main detection of the CYP2C19 gene was the 681 st locus on the fifth exon. The population could be divided into the wild type group (GG) and the mutant group (GA/AA) according to the different genotypes.

Statistical analysis was performed using SPSS v. 17.0 (SPSS, Inc., Chicago, IL). Statistical analysis was performed using the SPSS 13.0 software package (SPSS, Chicago, IL, USA). The genotype frequency distribution was tested for Hardy-Weinberg equilibrium (HWE) with a chi-square test. Differences of continuous variables with a normal distribution (presented as mean $\pm \mathrm{SD}$ ) between the two groups were calculated using the independent-sample t-test. Two-tailed $P$ values $<0.05$ were considered statistically significant. The relevance between CYP2C19 gene polymorphism, as well as other risk factors and coronary heart disease was tested by multiple linear regression analysis.

\section{Result}

1. The gene distribution of the study population conformed to the Hardy-Weinberg equilibrium [4] $\left(\chi^{2}=0.735, P=0.392\right)$, which means the study population was typical.

2. The clinical characteristics of the study individuals are summarized in Table 1. A chi-square test showed that the clinical characteristics, such as gender, diabetes, hypertension, hyperlipemia, overweight, and smoking, did not differ significantly between patients with different genotypes. A t-test showed that there was no significant difference in age between the two groups.

Table 1. The clinical characteristics of the study individuals

\begin{tabular}{lcccc}
\hline \multicolumn{1}{c}{ Variables } & $\begin{array}{c}\text { GA/AA group } \\
(\mathrm{n}=139)\end{array}$ & $\begin{array}{c}\text { GG group } \\
(\mathrm{n}=213)\end{array}$ & statistics & $P$ \\
\hline Gender (male), $\mathrm{n}$ & 89 & 130 & 0.321 & 0.653 \\
BMI>25kg/m², $\mathrm{n}$ & 73 & 106 & 0.255 & 0.663 \\
Smoking habit, $\mathrm{n}$ & 72 & 104 & 0.297 & 0.663 \\
Hyperlipidemia, $\mathrm{n}$ & 26 & 24 & 3.818 & 0.061 \\
Hypertension, $\mathrm{n}$ & 86 & 146 & 1.667 & 0.207 \\
Diabetes, $\mathrm{n}$ & 76 & 99 & 2.261 & 0.156 \\
Age, years & $66.30 \pm 8.63$ & $67.11 \pm 8.53$ & 0.867 & 0.387 \\
\hline
\end{tabular}

3. The age of onset of the individuals is summarized in Graph 1 and Tables 2 and 3. According to the study of Framingham, premature coronary heart disease was identified as CHD that occurs in males under 55 years of age and in females under 66 years of age. Graph 1 shows that there is a significantly different genotype distribution of the CYP2C19 $681 \mathrm{G}>\mathrm{A}$ polymorphism in different groups with different ages of onset. The frequency of the CYP2C19 GA/AA 
genotype was $0.480,0.503$ in patients with CHD who had an earlier age of onset and 0.324 in patients with CHD who had a later age of onset. A significant difference in the CYP2C19 $681 \mathrm{G}>\mathrm{A}$ genotype was observed in the different groups as shown in Table $2\left(\chi^{2}=10.753, P=0.005\right)$. The allele frequencies of the CYP2C19 A allele in patients with onset at $<40,40$ to 55 , and $>55$ years were $0.320,0.268$ and 0.227 , respectively. And there was a significant difference between the different groups $\left(\chi^{2}=10.745, P=0.005\right)$ as shown in Table 2 . The t-test showed that the age of onset in the mutant group was less than in the other group, $53.95 \pm 11.63$ years and $58.51 \pm 12.72$ years, respectively, as shown in Graph $2(t=3.398, P=0.001)$. And the difference was significant between the wild type and mutant groups as shown in Graph 2. Furthermore, after adjustment for traditional risk factors of CHD, CYP2C19 681A was significantly associated with the age of onset of patients with CHD by multiple linear regression analysis $(F=5.966, P=0.000)$ (Table 3$)$, as well as smoking, overweight and hyperlipidemia, and $\beta$ was $0.167,0.156,0.155$, and 0.122 . All of the risk factors can lead to an earlier onset of CHD.

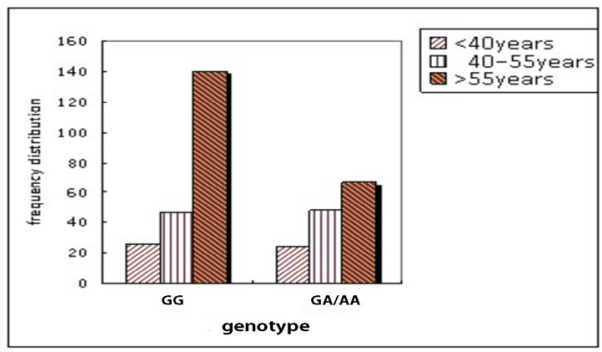

Graph 1. Genotype distribution of the CYP2C19 G681A polymorphism in different groups

Table 2. Genotype distribution of the CYP2C19 G681A polymorphism in different groups

\begin{tabular}{cccccc}
\hline Gene & $<40$ years & $40-55$ years & $>55$ years & $X^{2}$ & $P$ \\
\hline Group & & & & & \\
GA/AA & $24(48.0 \%)$ & $48(50.5 \%)$ & $67(32.4 \%)$ & 10.753 & 0.005 \\
GG & $26(52.0 \%)$ & $26(52.0 \%)$ & $140(67.7 \%)$ & & \\
Genotype & & & & & \\
AA & $8(16.0 \%)$ & $3(3.2 \%)$ & $10(4.8 \%)$ & & \\
GA & $16(32.0 \%)$ & $45(47.4 \%)$ & $57(27.5 \%)$ & 8.647 & 0.013 \\
GG & $26(52.0 \%)$ & $47(49.5 \%)$ & $140(67.7 \%)$ & & \\
Allele & & & & & \\
A & 0.32 & 0.268 & 0.227 & 10.745 & 0.005 \\
G & 0.68 & 0.732 & 0.773 & & \\
\hline
\end{tabular}

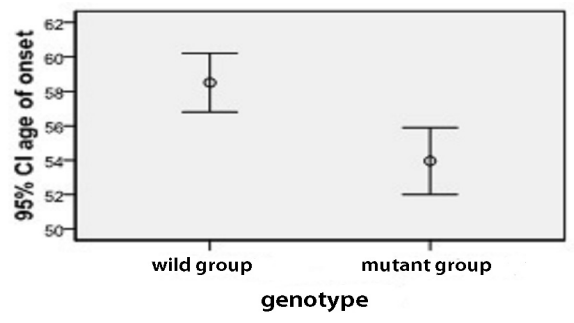

Graph 2. The difference* of age of onset with different genotype ( $*=3.398, P=0.001$ )
Table 3. Multiple linear regression analysis of determinants of CHD in individuals

\begin{tabular}{cccc}
\hline & $t$ & $\beta$ & $P$ \\
\hline Genotype & -3.244 & -0.167 & 0.001 \\
BMI $>25 \mathrm{~kg} / \mathrm{m}^{2}$ & 2.345 & 0.155 & 0.020 \\
Smoking & 2.007 & 0.156 & 0.046 \\
Hyperlipidemia & 2.174 & 0.112 & 0.030 \\
Diabetes & -0.835 & -0.071 & 0.404 \\
Hypertension & 0.936 & 0.048 & 0.350 \\
Constant & 8.85 & 44.557 & 0.000 \\
\hline
\end{tabular}

\section{Discussion}

CHD is one of the most serious diseases worldwide, endangering human health, and is a multifactorial and polygenic disease [5]. In recent years, the morbidity and mortality resulting from $\mathrm{CHD}$ has increased year by year. Moreover, its incidence rate among young patients is increasing. As estimated, there were 7.3 million deaths worldwide owing to CHD in 2001 [6]. With the development of molecular biological technology, more and more genetic research focuses on the pathogenesis of CHD. Studies have reported that a variety of genes are closely associated with CHD and other cardiovascular diseases, including the endothelial nitric oxide synthase gene, the angiotensin converting enzyme gene, and apolipoprotein E (APOE) [7-9]. A series of studies on genome-wide associations of CHD are ongoing, which are important to an understanding of the molecular and genetic pathogenesis of $\mathrm{CHD}$, as well as individual susceptibility to CHD. These studies are also important for achieving targeted or individual therapies so as to prevent the occurrence of CHD and improve the survival rates of patients with the disease. Multiple studies have shown that cytochrome P450, a super family of cysteinate-haem enzymes, plays an important role in the onset, progression, and prognosis of CHD [10]. Some researchers have shown that CYP2C $9 * 1 / * 3$ may be associated with premature coronary heart disease in Chinese Han males; similarly, CYP2J2*7 may be associated with premature coronary heart disease in Chinese Han women [11]. Some studies [12] have estimated that CYP3A4*1G/*1G may increase the risk of CHD in Chinese Han females. A large number of studies [13-14] in the relevant field from home and abroad have shown that CYP2C19*1/*2, *1/*3 with only one effective allele leads to enzyme activity reduction. Thus, the residual platelet aggregation rate in patients with CYP2C19*2 or *3 alleles is higher than that of the wild homozygous type. Furthermore, anti-platelet drug efficacy in patients with CYP2C19*2 and $* 3$ alleles is poorer, which can increase the incidence of adverse cardiovascular events [3].

With age of onset in patients with CHD as a focal point, our study observed the genotype distribution of the CYP2C19 $681 \mathrm{G}>\mathrm{A}$ polymorphism. The goal of this study was to evaluate the association of CYP2C19 polymorphism with CHD. The study showed that the frequency of the CYP2C19 GA/AA genotype was significantly higher in patients with earlier onset 
than in those with later onset $\left(\chi^{2}=8.647, P<0.001\right): 0.48$ in the $<40$ group, 0.505 in the 40 to 55 group, and 0.324 in the $>55$ group. In addition, the frequency of mutant allele was also significantly different in groups with the same 3 different ages of onset $\left(\chi^{2}=10.745, P=0.005\right): 0.32,0.268$ and 0.227 , respectively, as shown in Table 2. Graph 1 demonstrates that the distribution of earlier age of onset was different in patients with different CYP2C19 genotypes, and was bigger in the mutant group than in the wild type group. These results reveal that the CYP 2 C19 $681 \mathrm{G}>\mathrm{A}$ polymorphism might be associated with age of onset of CHD. Furthermore, the t-test in Graph 2 shows that the age of onset in patients with different CYP2C19 genotypes was significantly different $(t=3.398, P=0.001): 53.95 \pm 11.63$ years in the wild type group and $58.51 \pm 12.72$ years in the mutant group. After further analysis of the data, multiple linear regression found that genotype, smoking, overweight and hyperlipidemia are correlated with the age of onset of CHD $(\beta=-0.167$, $0.156,0.155$, and $0.112 ; P=0.001,0.046,0.020,0.030$ ); CYP $2 C 19681 \mathrm{G}>\mathrm{A}$ had the greatest effect on the age of onset, which indicates that $\mathrm{CYP} 2 \mathrm{C} 19$ gene polymorphism might not be an independent risk factor for $\mathrm{CHD}$; rather, there might be several reasons why CYP2C19 gene polymorphism is associated with CHD. Some studies have indicated that certain CYP enzymes play an important role in CHD progression, which is likely related to their character as major regulators of L-type calcium channels and cardiomyocyte contractility [15]. CYP2C19 and CYP2A1 are related to elevation of pro-inflammatory cytokines in heart failure [16]. CYP may also influence the pathogenesis of cardiac hypertrophy by enhancing the production of ROS [17]. In recent years, some scholars [18] have researched the effect of CYP450 family gene polymorphism in the metabolism pathways of arachidonic acid. They found that the CYP2 family expressed in the cardiovascular system and CYP2C19 is the key enzyme in the metabolism of arachidonic acid, which is relevant to endothelium-dependent vasodilation and plays an important role in regulation of the cardiovascular system. It is speculated that CYP2C19 $681 \mathrm{G}>\mathrm{A}$ polymorphism can impact the pathogenesis of CHD by reducing the enzyme activity. In addition, Giusti B found that the CYP2C19 gene polymorphism is relevant to platelet hyper reactivity in a study involving 1419 patients with CHD. This gene polymorphism causes the individual difference in endogenous and exogenous drug metabolism, and even some medical conditions such as cancer and birth defects, by leading the change in CYP2C19 protease activity [19]. Some documents [20] reported that having firstdegree relatives with a history of CHD not only increases the risk of CHD, but also influences the onset of CHD. Our study suggests that gene polymorphism is more likely to affect young rather than old patients and is affecting more young patients, which is consistent with some other previous studies [21]. Our study could provide new targets and ideas for the effective prevention and treatment of coronary heart disease.

In addition, our study also found that conventional risk factors, including, overweight, smoking and hyperlipidemia, are associated with the age of onset of CHD, as shown in Table 3, which is consistent with current research. The increase in coagulation factor activity in overweight patients can lead to enhanced platelet aggregation, which may be a possible cause of CHD in advance of the onset age. Smoking can cause vascular endothelial cell injury, which can result in coronary artery spasm or inflammation, even the formation of atherosclerotic plaque and CHD. Previous studies have demonstrated that lipid metabolic disorder is an independent risk factor for CHD and that the apolipoprotein E (APOE) gene is a susceptible CHD gene. A rise in triglyceride levels can increase risk of CHD by putting an individual in a hypercoagulable state. Our study shows that diabetes and hypertension are not relevant to the onset age of CHD, which is not consistent with previous studies [22]. We considered that the point of our study was the age of onset of CHD, but hypertension and diabetes are age-related diseases, which have less influence on patients with early age of onset.

Some research has shown that there are other types of mutations of CYP2C19, but we did not investigate that due to the low frequency of occurrence in the East Asian populations. Although other members of the CYP superfamily have been well studied regarding the onset, progression, and prognosis of CHD [17], little information is available about CYP2C19 specifically contributing to the pathogenesis of CHD, a subject that needs to be further studied. Since the study was a retrospective study, and subject to geographical constraints, we only provided the CYP $2 C 19681 \mathrm{G}>\mathrm{A}$ genotype distribution of Han populations in North China. What is more, age of onset is affected by many factors, which need more epidemiological studies in different areas and races with larger samples to estimate the relationship between CYP2C19 681G>A polymorphism and CHD.

In conclusion, we demonstrated a significant association between CYP2C19 681G>A polymorphism and age of onset of CHD in Han populations in North China, and the result also indicate that $C Y P 2 C 19681 \mathrm{G}>\mathrm{A}$ may be a gene polymorphism that is susceptible to CHD in the North Chinese Han population. Thus, we can improve the prevention and treatment of CHD by aiming at the susceptibility gene. For the conventional risks, including smoking, overweight and hyperlipidemia, we suggest that regular health examinations are essential for all individuals and that more attention be given to strengthening the primary prevention of CHD [23].

\section{Competing interests}

The authors declare that they have no competing interests.

\section{References}

1. Zhou X, Zhang JZ, Qi HZ, et al. Progress in cardiovascular drug metabolic enzyme and pharmacogenetic studies. China Pharm 2012; 23(26):2476-2479.

2. Shu Y, Zhou HH. Individual and ethnic differences in CYP2C19 activity in Chinese populations. Acta Pharmacol Sin 2000; 21(3): 193-9.

3. Ercan B, Ayaz L, Ciçek D, Tamer L.. Role of CYP2C9 and CYP2C19 polymorphisms in patients with atherosclerosis. Cell Biochem Funct 2008; 26 (3):309-13.

4. Motulsky V. Human genetics. The third edition. Beijing; 
People's Medical Publishing House 1999:749-750.

5. Gaziano TA, Bitton A, Anand S, Abrahams-Gessel S, Murphy A. Growing epidemic of coronary heart disease in low- and middle- income countries. Curr Probl Cardiol 2010; 35(2):72-115.

6. Kastelein JJ, van Leuven SI, Burgess L, Evans GW, Kuivenhoven JA, Barter PJ, et al. Effect of torcetrapib on carotid atherosclerosis in familial hypercholesterolemia. N Eng1 J Med 2007; 356(16): 1620-30.

7. Zhou B, Wu KX, Li Y, et al. Analysis of 236 cases of coronary angiography in patients with clinical background information. Chin J Prac Diag Treat,2008; 22(11):872-3.

8. Han Y, Xu W, Zhang W, Liu N, Ji Y.. T-786C polymorphism in the endothelial nitric oxide synthase gene is associated with increased risk of coronary artery disease in a Chinese population. Pharmacology 2010; 85(4):211-6.

9. Kolovou GD, Anagnostopoulou KK. Apolipoprotein E polymorphism, age and coronary heart disease. Ageing Res Rev2007; 6(2): 94-108.

10. Elbekai RH, EI-Kadi AO. Cytochrome P450 enzymes:central players in cardiovascular health and disease. Pharmacol Ther 2006; 112(2):564-87.

11. Zhan TY, Shi Lei, Zhao SJ, et al. The correlation between the CYP2J2* 7 gene polymorphism and coronary heart disease in Chinese Han population. Guangdong Med J 2011; 32(12):1543-5.

12. He BX, Shi L, Qiu J, Tao L, Li R, Yang L, Zhao SJ. A functional polymorphism in the CYP3A4 gene is associated with increased risk of coronary heart disease in the Chinese Han population. Basic Clin Pharmacol Toxicol 2011;108(3): 208-13.

13. Hulot JS, Bura A, Villard E, Azizi M, Remones V, Goyenvalle C, et al. Cytochrome P450 2C19 loss-offunction polymorphism is a major determinant of clopidogrel responsiveness in healthy subjects. Blood 2006; 108(7):2244-7. 14. Trenk D, Hochholzer W, Fromm MF, Chialda LE,
Pahl A, Valina CM, et al. Cytochrome P450 2C19 681G>A polymorphism and high on-clopidogrel platelet reactivity associated with adverse 1-year clinical outcome of elective percutaneous coronary intervention with drug-eluting or baremetal stents. J Am Coll Cardiol 2008; 51(20):1925-34.

15. Xiao YF, Huang L, Morgan JP. Cytochrome P450: a novel system modulating $\mathrm{Ca} 2+$ channels and contraction in mammalian heart cells. J Physiol 1998;508(Pt3):777-92.

16. Frye RF, Schneider VM, Frye CS, Feldman AM. Plasma levels of TNF-alpha and IL-6 are inversely related to cytochrome $\mathrm{P} 450$ dependent drug metabolism in patients with congestive heart failure. J Card Fail 2002; 8(5):315-9.

17. Elbekai RH, El-Kadi AO. Cytochrome P450 enzymes: central players in cardiovascular health and disease. Pharmacol Ther, 2006, 112(2):564-87.

18. Bièche I, Narjoz C, Asselah T, Vacher S, Marcellin P, Lidereau R, et al. Reverse transcriptase-PCR quantification of mRNA levels from cytochrome (CYP)1, CYP2 and CYP3 families in 22 different human tissues. Pharmacogenet Genomics, 2007 ; 17(9):731-42.

19. Wang Q, Zhang GJ, Kang XX. Effects of CYP2C19 gene polymorphisms on clopidogrel metabolism [J/CD]. Chin J Clin (Electronic Edition). 2013;7(21):9746-9.

20. Kral BG, Becker DM, Vaidya D, Yanek LR, Becker LC. Severity of inducible myocardial ischemia predicts incident acute coronary syndromes in asymptomatic individuals with a family hospital of premature coronary artery disease. J Nucl Cardiol 2012; 19(1):28-36.

21. Chu YR, Chu ZH, Zhu YL, Research on the relationship between apolipoprotein $\mathrm{E}$ gene polymorphism and early-onset coronary heart disease. Prog Mod Biomed 2007; 7(2):244-6.

22. Sun JG, Zhang XL, Hu JP. Sexual difference of clinical characteristics in premature coronary heart disease patients. Anhui Med J 2011; 32(2):213-5.

23. Yang JL, Yang FY. Risk factors for coronary heart disease and Prevention. PJCCPVD 2010; 18(7):1014-5. 